\title{
A Dual-Band MAC Protocol for Indoor Cognitive Radio Networks: An e-Health Case Study
}

\author{
Raúl Chávez-Santiago, llangko Balasingham \\ The Intervention Centre, Oslo University Hospital \\ Institute of Clinical Medicine, University of Oslo \\ Norwegian University of Science and Technology \\ $+(47) 23070123$ \\ raul.chavez-santiago@rr-research.no
}

\author{
Dainius Jankūnas, Vladislav V. Fomin \\ Vytautas Magnus University \\ Faculty of Informatics \\ 44249 Kaunas, Lithuania \\ dainiusneo@gmail.com
}

\begin{abstract}
The importance of wireless technology in modern medicine has increased in the last years. It is anticipated that a large number of wireless communication devices for e-health will operate in unlicensed frequency bands in indoor environments. This represents a coexistence problem, which will be particularly challenging in confined areas of hospitals. Electromagnetic interference (EMI) from wireless devices can disrupt the performance of non-communication electronic medical equipment. Cognitive radio is a technology that can ease the coexistence by protecting non-communication electronic medical equipment. In this work we improved a cognitive radio EMIaware protocol for e-health applications. The original protocol protects medical equipment from harmful interference by preventing wireless transmissions when interference immunity levels are exceeded. However, this leads to high outage probability in areas where protected medical apparatuses are located. In order to maintain a low outage probability under this scheme, we propose the use of an additional channel in a different frequency band for control/data transmission from potential interference sources. We considered the recently allocated 2360-2400 MHz for medical body area networks and the 902-928 MHz band for allocation of the additional control/data channel. Simulation results demonstrated that the use of the proposed dual-band EMI-aware protocol using the $902-928 \mathrm{MHz}$ band significantly reduces the outage probability.
\end{abstract}

\section{Categories and Subject Descriptors}

H.4.3 [Information Systems Applications]: Communications Applications - computer conferencing, teleconferencing, and videoconferencing

\section{General Terms}

Design, Reliability, Verification.

\section{Keywords}

Cognitive radio, Indoor propagation, MAC layer, Outage.

Permission to make digital or hard copies of all or part of this work for personal or classroom use is granted without fee provided that copies are not made or distributed for profit or commercial advantage and that copies bear this notice and the full citation on the first page. To copy otherwise, to republish, to post on servers or to redistribute to lists, requires prior specific permission and/or a fee.

PFT 2013, September 30-October 02 Copyright @ $\odot 2013$ ICST 978-1-936968-89-3

DOI 10.4108/icst.bodynets.2013.253619

\section{INTRODUCTION}

The role of wireless communication technology in healthcare and medicine has gained significant importance in recent years [1]. Monitoring of physiological signals through the use of biomedical sensors equipped with wireless transceivers has become possible. For instance, small IEEE 802.15.4-compliant transceivers operating in the $2.4 \mathrm{GHz}$ industrial, scientific, and medical (ISM) band are suitable for medical sensing and are commercially available. Moreover, the IEEE 802.15.6 standard for wireless body area networks (WBANs) has also identified the $2.4 \mathrm{GHz}$ ISM band for the operation of wearable medical wireless sensors. In addition, a large number of ubiquitous IEEE 802.11 networks operate in $2.4 \mathrm{GHz}$. Therefore, it is anticipated that a large number of wireless communication devices operating in the 2.4 $\mathrm{GHz}$ ISM will coexist in hospital premises. Techniques to avoid mutual interference must be applied. The coexistence problem will be even more challenging in confined areas like intensive care units (ICUs) and operating rooms (ORs), because electromagnetic interference (EMI) from wireless devices can disrupt the performance of non-communication electronic medical equipment that is routinely present in these areas. Cognitive radio (CR) is a promising technology that can ease the coexistence of wireless devices while protecting medical equipment in hospitals [2], [3]. More specifically, in [4] a CR request-to-send/clear-tosend (RTS/CTS) protocol for e-health applications in hospitals was proposed. This protocol adapts the transmit power of wireless devices operating in $2.4 \mathrm{GHz}$ according to standardized EMI susceptibility constraints. In addition, the protocol differentiates between two types of medical applications with different priorities. Through computer simulations it was demonstrated that this EMI-aware RTS/CTS protocol can reduce significantly the interference to protected medical devices in comparison to a traditional RTS/CTS protocol; however, this comes at the expense of high outage probability for the wireless devices in areas where protected medical equipment is located. Hence, we set out to improve the EMI-aware RTS/CTS protocol in [4] by including dual-band operation. We considered a system operating in the 2.4 GHz ISM band with two channels, i.e., a control channel and a data channel. We propose the use of an additional "emergency" channel in a different frequency band that can serve as a control/data channel for potential interferers in order to reduce the outage probability. For this sake, we considered the 2360-2400 $\mathrm{MHz}$ frequency band and the 902-928 MHz ISM band. Through computer simulations we evaluated the performance of this multiband medium access control (MAC) scheme in terms of the outage probability and compared it to the original EMI-aware RTS/CTS protocol in [4]. Clearly, the use of an additional channel reduced the outage probability. However, the obtained level of improvement was determined by the operation frequency 
of the additional channel. Marginal improvement was obtained with an additional channel in the $2360-2400 \mathrm{MHz}$ frequency band, whereas significant improvement was obtained by using a channel in the 902-928 MHz ISM band.

The rest of the paper is organized as follows: In Section 2 we describe the CR system for hospital environments that we used as a basis for further development and improvement. Section 3 presents our proposed dual-band EMI-aware RTS/CTS protocol and its evaluation through computer simulations. In Section 4 we discuss our results and summarize our conclusions.

\section{EMI-AWARE RTS/CTS CHANNEL ACCESS MECHANISM}

\subsection{Architecture of the Cognitive Radio}

\section{System}

Two different types of e-heath applications were considered in [4], namely real-time non-critical telemedicine and a hospital information system. The telemedicine system is used to transmit data that are not delay/loss-sensitive, e.g., remote consultation, patient record transfers, and remote diagnosis. The hospital information system applications collect patient, technical, and facility data, which are intended for better clinical decisions and to prevent patient complications. In the $\mathrm{CR}$ context, the telemedicine system was treated as primary user (PU) and the hospital information system as secondary user (SU).

The CR system consists of three components, namely an inventory system, a CR controller, and CR clients. The inventory system is basically a database containing information about the medical devices in the hospital premises. Information like location, activity status, and EMI immunity levels are stored. Although in [4] it was proposed to use radio frequency identification (RFID) technology for tracking of the medical devices, it is important to consider that it has been reported that harmful interference from passive RFID disrupted critical care medical equipment in controlled experiments [5]. Therefore, alternative tracking technologies [6] should be considered for this scenario. The CR controller (CRC) controls the transmission parameters of the $\mathrm{CR}$ clients, i.e., PUs and SUs. For this sake, the CRC uses the information in the inventory system to compute the appropriate transmit power for each $\mathrm{CR}$ client in order to avoid exceeding the EMI immunity levels of non-communication medical devices located in the vicinity.

\subsection{Channel Access}

The CR system operates using a dedicated control channel (DCC) and a data channel. Both channels are in unlicensed spectrum, in this case the $2.4 \mathrm{GHz}$ ISM band. Every CR client transmits its data through the CRC. It was assumed that the CRC can transmit/receive data from both channels simultaneously, whereas the CR clients can transmit in one of the two channels at a time.

In order to access the channel, a time slotted RTS/CTS-based channel access mechanism is used by PUs and SUs. This consists of two steps: common control broadcasting and EMI-aware RTS/CTS protocol. The flowchart of this mechanism can be found in [4].

The DCC is used to broadcast information about the maximum power, $P_{\text {ctrl }}$, for transmitting the RTS message by each of the CR clients. Each $\mathrm{CR}$ client has a different $P_{\text {ctrl }}$ depending on its location, and it is calculated as

$$
P_{\text {ctrl }}=\min \left\{\min _{n}\left(P_{\mathrm{NLS}}(n)\right), \min _{m}\left(\mathrm{P}_{\mathrm{LS}}(m)\right)\right\}
$$

where $P_{\mathrm{NLS}}(n)$ and $P_{\mathrm{LS}}(m)$ are the upper bounds on transmit power for non-life-supporting (NLS) medical device $n$ and life-supporting (LS) medical device $m$, respectively. For a frequency range of $800-2500 \mathrm{MHz}$, these transmit powers can be computed as

$$
P_{\mathrm{NLS}}(n)=\left(\frac{D_{\mathrm{NLS}}(n) E_{\mathrm{NLS}}(n)}{7}\right)^{2}
$$

and

$$
P_{\mathrm{LS}}(m)=\left(\frac{D_{\mathrm{LS}}(m) E_{\mathrm{LS}}(m)}{23}\right)^{2}
$$

where $D_{\mathrm{NLS}}(n)$ and $D_{\mathrm{LS}}(m)$ are the distances from the CR client to the NLS device $n$ and LS device $m$, respectively. $E_{\mathrm{NLS}}(n)$ and $E_{\mathrm{LS}}(m)$ are the EMI immunity levels for the NLS and LS medical devices $n$ and $m$, respectively, the values of which are stored in the inventory system. Since the protected non-communication medical devices can be turned ON or OFF, and the locations of the CR clients change dynamically, $P_{\text {ctrl }}$ is computed and broadcast every $t_{p}$ slots on the DCC. All the transmissions from CR clients are paused during broadcasting of $P_{\text {ctrl }}$ to synchronize with the CRC.

In order to access the channel, a CR client transmits a RTS message to the CRC on the DCC. If a collision occurs, the colliding CR clients wait for a random time based on a constant backoff window for PUs and exponential backoff window for SUs. The CR clients can retransmit the RTS message with probability $\alpha_{1}$ for PUs and $\alpha_{2}$ for SUs. The number of SUs that can be in a queue (referred to as imaginary orbit) waiting for retransmission are limited, whereas it is infinite for PUs. When a RTS is successfully received by the CRC, the maximum transmit power on the data channel, $P_{\text {data }}$, is computed in the same way as $P_{\text {ctrl }}$. If the CRC cannot find a suitable transmit power that satisfies the minimum quality of service (QoS) of the CR client without violating the EMI constraints given by (2) or (3), the request for data transmission is dropped. In addition, the CRC randomly drops RTS messages with probability $P_{d 1}$ for PUs and $P_{d 2}$ for SUs in order to avoid congestion. If the CR client's RTS is dropped, a negative-CTS message is sent by the CRC; after a random number of time slots the $\mathrm{CR}$ client can attempt to transmit again. If the RC client is not dropped, then a CTS message is sent. After the CTS message is successfully received by the CR client, this will wait in the transmission queue. PUs and SUs wait in separated queues of finite size, which means a CR client's request will be dropped if the queue is full. PUs always have priority to transmit on the data channel. The number of time slots for data transmission was assumed to be geometrically distributed with parameters $\beta_{1}$ and $\beta_{2}$ for PUs and SUs, respectively.

\subsection{Performance Evaluation}

The EMI-aware protocol described above was evaluated through numerical simulations in [4] in terms of interference probability 
and outage probability. We reproduced the results reported therein in order to have a basis for fair comparison and assessment of our subsequent proposed improvements. The simulation scenario consisted of hospital premises over $27 \mathrm{~m}^{2}$ arranged in nine areas of equal size comprising a hall way, an administration room, and five ICUs as illustrated in Figure 1. The CRC was located at the center of area 5. Ten NLS and LS non-communication medical devices were located in the ICUs, and their corresponding EMI immunity levels are given in Figure 1. The locations of the NLS and LS medical devices and the CRC were fixed, whereas the CR clients were uniformly distributed over the area. We included a random mobility model for the $\mathrm{CR}$ clients to mimic wandering of the CR clients over the nine areas. In order to compute (2) and (3), the following indoor path loss (PL) formula as a function of the distance, $d(d>1)$, was applied

$$
\mathrm{PL}_{\text {total }}=37.7+3.3 \log _{10}(d)+16.2 n
$$

where $n$ is the number of floors (or walls) the radio signal has to traverse. The interference probability was defined as the chance that a wireless $\mathrm{CR}$ client causes interference to the noncommunication medical devices by violating (2) or (3). In the EMI-aware RTS/CTS protocol, interference occurs when the ON status of the medical devices is reported wrongly to the CRC. This probability of misdetection was assumed equal to 0.01 .

First, we ran simulations similar to those in [4] and obtained the interference probability of each area in Fig. 1. The results were compared to the traditional carrier sense multiple access with collision avoidance (CSMA/CA) RTS/CTS protocol. Figure 2 shows the simulation results. As seen, the EMI-aware RTS/CTS protocol successfully protected NLS and LS medical devices. These results are in agreement with what is reported in [4].

We also reproduced and compared the outage probability for both traditional CSMA/CA and EMI-aware RTS/CTS protocols. For this sake, the probability that the received signal strength at the $\mathrm{CRC}$ is less than $-65 \mathrm{dBm}$ was computed. Figure 3 shows the simulation results, which are also consistent with [4]. The parameters used in the aforementioned simulations are summarized in Table 1.

\begin{tabular}{|c|c|c|}
\hline $\begin{array}{l}\begin{array}{c}\text { Inventory } \\
\text { System }\end{array} \\
\text { Area } 7 \\
\text { Admin. Room }\end{array}$ & $\begin{array}{l}\qquad \begin{array}{l}\boldsymbol{E}_{\mathrm{NLS}}=\mathbf{3} \\
\boldsymbol{E}_{\mathrm{NLS}}=\mathbf{2}\end{array} \\
\text { Area } 8 \\
\text { ICU } 5\end{array}$ & $\begin{array}{l}\quad \boldsymbol{E}_{\mathrm{NLS}}=\mathbf{4} \\
\quad \boldsymbol{E}_{\mathrm{LS}}=\mathbf{8}\end{array}$ \\
\hline $\begin{array}{l}\text { Area } 4 \\
\text { Hall way }\end{array}$ & $\begin{array}{l}\text { CRC } \\
\text { Area } 5 \\
\text { Hall way } \\
\end{array}$ & $\begin{array}{l}\quad \boldsymbol{E}_{\mathrm{NLS}}=\mathbf{5} \\
\boldsymbol{E}_{\mathrm{LS}}=\mathbf{1 0} \\
\text { Area 6 } \\
\text { ICU 3 }\end{array}$ \\
\hline $\begin{array}{l}\text { Area } 1 \\
\text { Hall way }\end{array}$ & $\begin{array}{l}\quad \begin{array}{r}\boldsymbol{E}_{\mathrm{NLS}}=5 \\
\boldsymbol{E}_{\mathrm{NLS}}=\mathbf{4}\end{array} \\
\text { Area 2 } \\
\text { ICU 1 }\end{array}$ & $\begin{array}{l}\quad \begin{array}{l}\boldsymbol{E}_{\mathrm{NLS}}=\mathbf{3} \\
\boldsymbol{E}_{\mathrm{LS}}=\mathbf{1 2}\end{array} \\
\text { Area 3 } \\
\text { ICU 2 }\end{array}$ \\
\hline
\end{tabular}

Figure 1. Hospital scenario used for simulations (see [4] for more details).

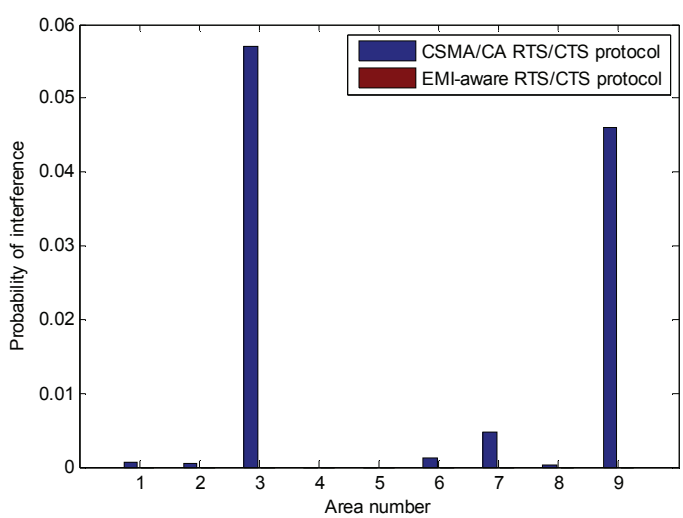

Figure 2. Interference probability over the nine areas.

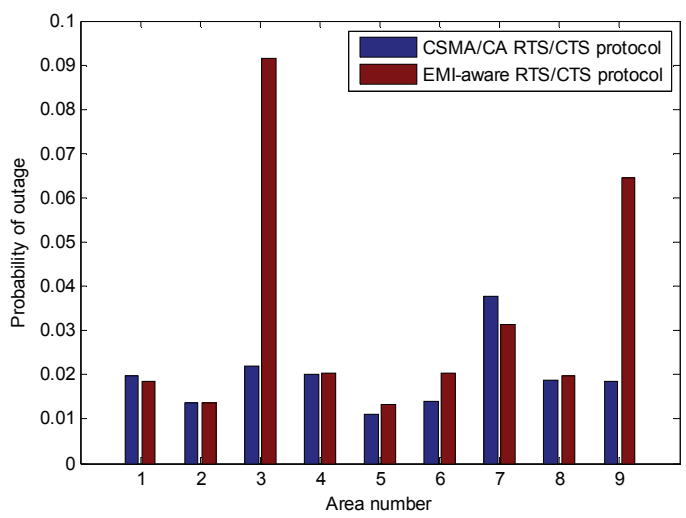

Figure 3. Outage probability over the nine areas.

Table 1. Parameters used for the computer simulations

\begin{tabular}{|l|c|}
\hline \multicolumn{1}{|c|}{ Simulation Parameter } & Value \\
\hline Bit rate & $250 \mathrm{Kbps}$ \\
\hline Traffic (max. waiting time) & $0.4 \mathrm{~s}$ \\
\hline Synchronization time with CRC & $0.1 \mathrm{~s}$ \\
\hline Slot Time & $20 \mu \mathrm{s}$ \\
\hline SIFS & $10 \mu \mathrm{s}$ \\
\hline DIFS & $50 \mu \mathrm{s}$ \\
\hline ACK message length & 14 bytes \\
\hline RTS message length & 20 bytes \\
\hline CTS message length & 14 bytes \\
\hline Data packet size & Max. 133 bytes \\
\hline Contention window (min. size) & 16 \\
\hline Contention window (max. size) & 256 \\
\hline Number of CR clients & 100 \\
\hline Motion speed of the CR clients & $0.5 \mathrm{~m} / \mathrm{s}$ \\
\hline Random drop probabilities & $P_{d 1}=0.3, P_{d 2}=0.4$ \\
\hline Size of the orbit & 3 \\
\hline Total simulation time & $120 \mathrm{~s}$ \\
\hline
\end{tabular}




\section{DUAL-BAND EMI-AWARE PROTOCOL}

We can see in Figure 3 that the outage probability in most areas for the EMI-aware RTS/CTS protocol is larger than it is for traditional CSMA/CA. Since the EMI-aware RTS/CTS protocol limits the transmit power of CR clients in order to protect medical devices in areas in which the EMI immunity values of (mainly LS) medical devices are high, e.g., areas 3 and 9, the transmission from some $\mathrm{CR}$ clients cannot reach the $\mathrm{CRC}$ with the minimum required signal strength. In other areas, however, the outage probability is practically the same for both protocols.

Therefore, to alleviate the outage probability problem we investigated the use of an additional channel to handle the CR clients' transmission requests that are dropped by the CRC because of violations of (2) or (3). Clearly, the selected channel must allow transmission with lower power while ensuring the received signal strength at the $\mathrm{CRC}$ is above $-65 \mathrm{dBm}$.

In indoor propagation scenarios the path loss is frequencydependent; let us, for instance, look at the ITU indoor path loss model [7], which is expressed as

$$
\mathrm{PL}_{\text {total }}=20 \log _{10}(f)+N \log _{10}(d)+L_{F}(n)-28 \mathrm{~dB}
$$

where $N$ is an empirical path loss exponent, $f$ is the signal frequency in $\mathrm{MHz}$, and $L_{F}(n)$ is the floor (or wall) penetration loss factor. Notice that (4) is a particular case of (5) for $f=2400 \mathrm{MHz}$. This model covers a wide range of frequencies, from $900 \mathrm{MHz}$ to $5.2 \mathrm{GHz}$. The values of $N$ and $L_{F}(n)$ for different frequencies can be found in [7]. It is evident that frequencies below $2.4 \mathrm{GHz}$ for the additional emergency channel result in lower path loss, which means that less transmit power is necessary to reach the CRC with the required signal strength.

We assumed that the DCC and data channel were in the $2.4 \mathrm{GHz}$ ISM band. Since IEEE 802.11 networks also operate in this band, we assumed that the DCC was centered at $2480 \mathrm{MHz}$ whereas the data channel was centered at $2475 \mathrm{MHz}$. We chose these frequencies because they do not overlap with the three most used non-overlapping IEEE 802.11 channels in the United States, namely channel 1 ( $\left.f_{c}=2412 \mathrm{MHz}\right)$, channel $6\left(f_{c}=2437 \mathrm{MHz}\right)$, and channel $11\left(f_{c}=2462 \mathrm{MHz}\right)$. Then, we considered two frequency bands for the implementation of the dual-band EMIaware mechanism. We first considered the 2360-2400 MHz frequency band, which was recently approved by the Federal Communications Commission (FCC) for medical body area network (MBAN) use on a secondary basis. In this case, we assumed that the additional channel was centered at $2365 \mathrm{MHz}$. We also considered the $902-928 \mathrm{MHz}$ ISM band, where the additional channel was assumed to be centered at $906 \mathrm{MHz}$.

In this dual-band EMI-aware RTS/CTS protocol, if a CR client wants to transmit data, first it sends a RTS message on the DCC to the CRC. If the CRC estimates that the CR client will not cause interference to the protected medical equipment, then the $\mathrm{CR}$ client is allowed to transmit its RTS message on the DCC. Otherwise, the CR client tries to switch to the additional channel (centered at $2365 \mathrm{MHz}$ or $906 \mathrm{MHz}$ ) for RTS transmission. The maximum transmit power on the additional channel is then calculated. If the maximum transmit power on the additional channel meets the minimum signal strength requirement without causing interference, the $\mathrm{CR}$ client is allowed to transmit data on this same additional channel. In this case, however, there is no transmission queue. Hence, before sending the RTS message on the additional channel the $\mathrm{CR}$ client has to ensure that said channel is free. If the additional channel is not free or a collision occurs, then the CR client's transmission request is dropped immediately. In the next section we will discuss the reason for this assumption. Both PUs and SUs can equally access the additional control/data channel. If only SUs were allowed, then there would be situations in which SUs have more probability of successfully transmitting their data than PUs.

We performed simulations of the proposed dual-band MAC scheme under similar traffic conditions for all the cases and the results are shown in Figure 4. As seen, little reduction of outage probability resulting from the use of an additional channel centered at $2365 \mathrm{MHz}$ can be observed in areas 3, 7, and 9, whereas in the other areas there is practically no improvement. This is expected since the spectral separation between the DCC $(2480 \mathrm{MHz})$ and the additional control/data channel $(2365 \mathrm{MHz})$ is relatively small. The improvement, however, is much more significant when the additional channel is centered at $906 \mathrm{MHz}$. In this case, a maximal outage probability reduction of $84.7 \%$ with respect to the scheme in [4] was obtained. Similar high improvement can be observed in area 3. In area 7, the achieved outage probability reduction was just $19.9 \%$. For the rest of the areas there was no significant improvement or no improvement at all. Finally, we investigated the performance of a multi-band MAC scheme in which two additional channels, one centered at $2365 \mathrm{MHz}$ and the other at $906 \mathrm{MHz}$, were used. In this scheme, if a CR client cannot transmit on the DCC, then it will attempt first to use the channel centered at $2365 \mathrm{MHz}$. If allowed, the CR client will transmit its data on this channel; otherwise, instead of being immediately dropped the CR client will be allowed to attempt transmission on the additional channel centered at 906 $\mathrm{MHz}$. Only if the CR client cannot transmit in the lowest frequency channel then its transmission request is dropped. As seen in Figure 4, this multi-band MAC scheme did not provide any significant improvement with respect to the dual-band scheme with an extra channel centered at $906 \mathrm{MHz}$. Therefore, the use of only one additional channel is recommended.

In all the above cases, the CR clients and the CRC must be implemented preferably on software-defined radio (SDR) platforms [8], [9] which allow easy reconfiguration of the communication interface parameters as required by the proposed dual-band EMI-aware MAC protocol.

\section{DISCUSSION AND CONCLUSIONS}

Multichannel MAC mechanisms have been previously studied in the literature [10]-[12], but in most of those cases the multiple channels are contiguous in frequency, i.e., in the same frequency band. Our study was aimed at obtaining insight into the behavior of dual-band RTS/CTS-based MAC schemes. As it was demonstrated by our simulations, using different frequency bands for the multiple channels of a cognitive MAC scheme can greatly help to alleviate the outage probability of an already existing EMI-aware RTS/CTS protocol for indoor cognitive networks. Although our work focused on the case study of a hospital with ehealth applications, the same concept of a dual-band EMI-aware RTS/CTS protocol can be extended to other indoor cognitive network scenarios. 


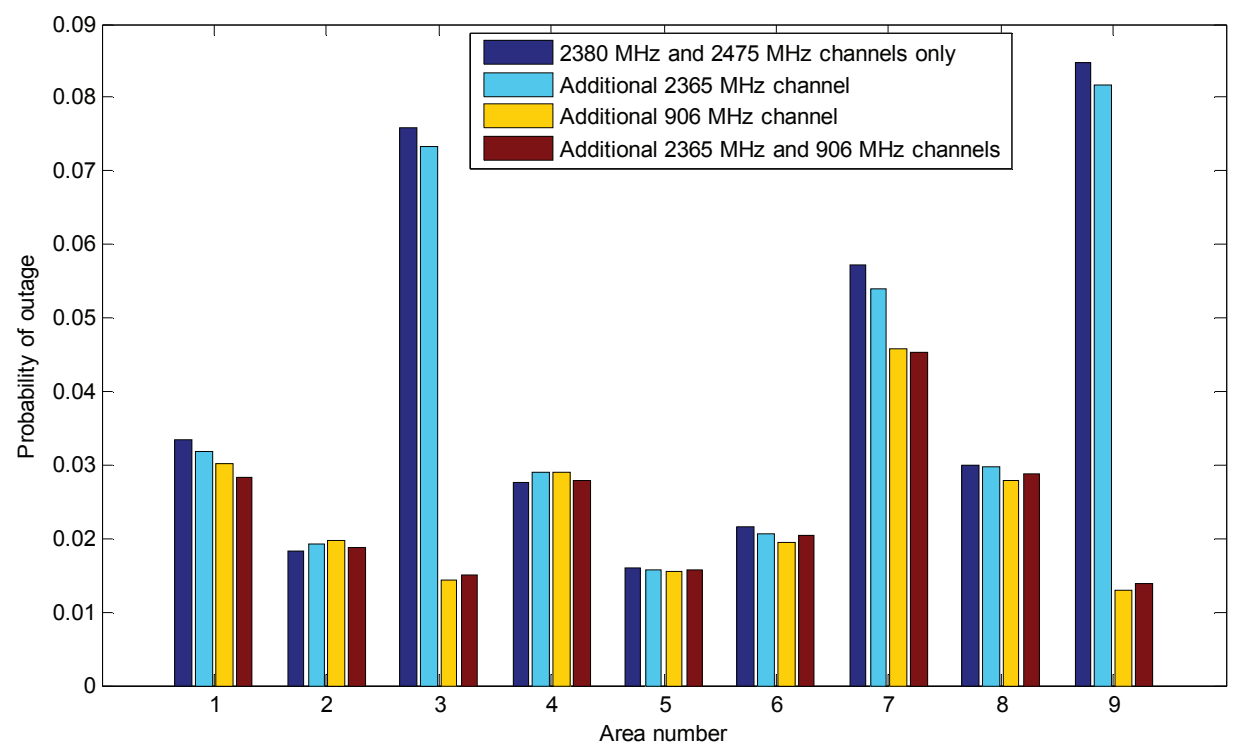

Figure 4. Outage probability over the nine areas for different multiband EMI-aware RTS/CTS protocols.

However, it is important to keep in mind that in a real cognitive network the additional control/data channel will not be reserved as in our simulations. In fact, this additional channel will be a spectrum hole detected dynamically by the CRC. This is why it is not possible to have a transmission queue for accessing this channel, since any transmission on it will be done opportunistically. To identify available spectrum holes in real time, the indoor area can be equipped with a sensor network for spectrum sensing as proposed in [13] in order to assist the CRC.

Our future simulations will consider a dynamic allocation of the emergency channel depending on real spectrum occupancy conditions. For this sake, a radio environment map (REM) will be constructed based on statistics obtained through spectrum measurement campaigns in hospital premises. Hence, the availability and operation frequency of the additional channel will vary over time. In this way, a more realistic performance of the proposed dual-band EMI-aware RTS/CTS protocol will be simulated. Moreover, other parameters of interest like throughput and delay will be assessed. Clearly, much remains to be done.

\section{ACKNOWLEDGMENTS}

This work is the result of ongoing international cooperation supported by the COST Action IC0905 TERRA (http://www.costterra.org). Partial funding for the research was provided to D. Jankūnas by the COST Action IC0905-TERRA through a Short Term Scientific Mission (Ref. COST-STSM-ECOST-STSMIC0905-150213-026948); additional funding was made available by ERASMUS. R. Chávez-Santiago and I. Balasingham acknowledge financial support from the Research Council of Norway provided through the MELODY-II Project (Contract no. 225885); additional funding came from Helse Sør-Øst Norway through the Innovation Grant no. 11/01137-156.

\section{REFERENCES}

[1] Alemdar, H., and Ersoy, C. 2010. Wireless sensor networks for healthcare: a survey. Computer Networks. 54, 15 (Oct. 2010), 2688-2710. DOI= http://dx.doi.org/10.1016/j.comnet.2010.05.003

[2] Doost-Mohammady, R., and Chowdhury, K. R. 2012. Transforming healthcare and medical telemetry through cognitive radio networks. IEEE Wireless Commun. 19, 4, (Aug. 2012), 67-73. DOI=

http://dx.doi.org/10.1109/MWC.2012.6272425

[3] Chávez-Santiago, R., Nolan, K. E., Holland, O., De Nardis, L., Ferro, J. M., Barroca, N., Borges, L. M., Velez, F. J., Goncalves, V., and Balasingham, I. 2012. Cognitive radio for medical body area networks using ultra wideband. IEEE Wireless Commun. 19, 4, (Aug. 2012), 74-81. DOI= http://dx.doi.org/10.1109/MWC.2012.6272426

[4] Phunchongharn, P., Hossain, E., Niyato, D., and Carmolinga, S. 2010. A cognitive radio system for e-health applications in a hospital environment. IEEE Wireless Commun. 17, 1, (Feb. 2010), 20-28. DOI= http://dx.doi.org/10.1109/MWC.2010.5416346

[5] Van der Togt, R., Van Lieshout, E. J., Hensbroek, R., Beinat, E., Binnekade, J. M., and Bakker, P. J. 2008.

Electromagnetic interference from radio frequency identification inducing potentially hazardous incidents in critical care medical equipment. JAMA. 299, 24, (Jun. 2008), 2884-2890. DOI= 10.1001/jama.299.24.2884

[6] Khoury, H. M., and Kamat, V. R. 2009. Evaluation of position tracking technologies for user localization in indoor construction environments. Automation in Construction. 18, 4, (Jul. 2009), 444-457. DOI= http://dx.doi.org/10.1016/j.autcon.2008.10.011 
[7] Seybold, J. S. 2005. Introduction to RF Propagation. Hoboken, John Wiley \& Sons Inc., NJ.

[8] Ulversøy, T. 2010. Software defined radio: challenges and opportunities. IEEE Commun. Surv. Tutor. 12, 4, (2010), $531-550 . \mathrm{DOI}=$

http://dx.doi.org/10.1109/SURV.2010.032910.00019

[9] Chávez-Santiago, R., Mateska, A., Chomu, K., Gavrilovska, L., and Balasingham, I. 2013. Applications of software defined radio (SDR) technology in hospital environments. In Proceedings of the 35th Annual International Conference of the IEEE Engineering in Medicine \& Biology Society (Osaka, Japan, July 3-7, 2013). EMBC '13.

[10] Han, Y. S., Deng, J., and Haas, Z. J. 2006. Analyzing multichannel medium access control schemes with ALOHA reservation. IEEE Trans. Wireless Commun. 5, 8, (Aug.
2006), 2143-2152. DOI=

http://dx.doi.org/10.1109/TWC.2006.1687730

[11] Deng, J., Han, Y. S., and Kulkarni, S. R. 2009. Can multiple subchannels improve the delay performance of RTS/CTSbased MAC schemes? IEEE Trans. Wireless Commun. 8, 4, (Apr. 2009), 1591-1596. DOI= http://dx.doi.org/10.1109/TWC.2009.060932

[12] De Domenico, A., Strinati, E. C., and Di Benedetto, M.-G. 2012. A survey on MAC strategies for cognitive radio networks. IEEE Commun. Surv. Tutor. 14, 1, (2012) 21-44. DOI= http://dx.doi.org/10.1109/SURV.2011.111510.00108

[13] Mercier, B., et al. 2008. Sensor networks for cognitive radio: theory and system design. In Proceeding. of the ICT Mobile and Wireless Communications Summit (Stockholm, Sweden, June 10-12, 2008). ICT MobileSummit '08. 